\title{
Efficacy of He-Ne Laser in Combination with Topical Clindamycin in the Treatment of Acne Vulgaris (AV)
}

\section{Bui Thi Van1, Nguyen Ngoc Oanh², Đặng Văn Em¹, Huynh Thi Xuan Tam³, Nguyen Lan Anh1, Pham Thi Minh Phuong²}

\author{
${ }^{1}$ Dermatology-Venereology and Allergy, 108 Central Military Hospital, Hanoi, Vietnam \\ ${ }^{2}$ Dermatology-Venereology Department, Bach Mai Hospital, Hanoi, Vietnam \\ ${ }^{3}$ Pham Ngoc Thach University of Medicine, Ho Chi Minh City, Vietnam \\ Email: tamhtx@pnt.edu.vn
}

How to cite this paper: Van, B.T., Oanh, N.N., Em, Đ.V., Tam, H.T.X., Anh, N.L. and Phuong, P.T.M. (2020) Efficacy of He-Ne Laser in Combination with Topical Clindamycin in the Treatment of Acne Vulgaris (AV). Journal of Cosmetics, Dermatological Sciences and Applications, 10, 134-139.

https://doi.org/10.4236/jcdsa.2020.103015

Received: July 15, 2020

Accepted: September 8, 2020

Published: September 11, 2020

Copyright $\odot 2020$ by author(s) and Scientific Research Publishing Inc. This work is licensed under the Creative Commons Attribution International License (CC BY 4.0).

http://creativecommons.org/licenses/by/4.0/

\begin{abstract}
Objective: To evaluate the efficacy of He-Ne laser in combination with topical clindamycin in the treatment of AV at 108 Military Central Hospital from Oct 2015 to Aug 2016. Subjects and Methods: 61 patients with AV were divided into 2 groups: Group 1: 31 patients were treated with He-Ne laser in combination with topical Clindamycin, Group 2: 30 patients were only treated with topical clindamycin. Laser HE-NE was applied 2 times/week for 6 weeks. Result: After 3 months of treatment Group 1: very good-51.6\%, good-48.4\%. Group 2: very good $-30 \%$, good $-50 \%$ and moderate $-20 \%$. Conclusion: The combination of He-Ne laser with topical clindamycin shows more rapid clinical improvement compared to topical clindamycin alone in the treatment of mild and moderate AV.
\end{abstract}

\section{Keywords}

Acne Vulgaris, He-Ne Laser, Clindamycin

\section{Introduction}

Acne vulgaris is a very common skin disease in both men and women with about $80 \%$ of adults. Especially, it most often affects adolescents from 13 to 25 years old [1] [2]. The pathogenic factors of AV hyperkeratosis might include infra-infundibulum, sebaceous duct, hyperactivity of sebaceous gland, hyperseborrhea, hyperproliferation of $P$. acnes, inflammation and immunological host reaction. In addition, factors such as family factors, psychological impact, environment, diet, personal hygiene also greatly affect the incidence of acne [3]. 
Clinical symptoms vary depending on the inflammation grades, increasing secretion and stagnation in sebaceous gland [4]. Most acnes do not cause serious complications, but the disease appearing mainly on the face would affect the aesthetic, psychology, confidence in communication, which deeply affects the patients' quality of life.

Helium-Neon laser ( $\mathrm{He}-\mathrm{Ne})$ is a low-energy laser used in clinical practice. The main effect of He-Ne laser is biological stimulation, enhancing organization, increasing metabolism, increasing microcirculation in the tissues, increasing protein synthesis and activating the immune system [2] [5]. Clindamycin is an antibiotic used in acne treatment.

Therefore, this study was conducted with the objective to evaluate the efficacy of He-Ne laser combined with topical clindamycin in the treatment of acne vulgaris.

\section{Subjects and Methods}

61 patients with mild to moderate acnes AV were selected from the Department of Dermato-venereology and Allergy of 108 Central Military Hospital from Oct 2015 to Aug 2016.

Inclusion criteria: Patients with mild and moderate acne vulgaris.

Exclusion criteria: Age $<12$ years, pregnancy, lactation, clindamycin allergy, light sensitivity. All patients were provided written informed consent.

This is a randomized, comparative and prospective study. 61 patients with acne vulgaris were divided into 2 groups: Group 1: 31 patients-He-Ne laser and topical clindamycin 1\%, Group 2: 30 patients-topical clindamycin simple. Laser He-Ne was applied 2 times/week for 6 weeks.

The measurement of the acne treatment results was based on the percentage reduction of total lesions, with 4 levels: Very good: comedones, papules decreased $\geq 75 \%$, pustules cleared; Good: comedones, papules decreased $50-<75 \%$, pustules cleared; Moderate: comedones, papules decreased $25-<50 \%$, pustules decreased; Poor: comedones, papules decreased $<25 \%$, pustules remained [3]. We photographed our patients at each time they visited and make a comparison between 4 timelines: at the beginning, after 1, 2, 3 months by counting the lesions.

The study was approved by The Board of Ethics in 108 Central Military Hospital at Ha Noi.

Data was analyzed by using SPSS software statistical computer package version 16. For quantitative data Student's t-test was used and the means and standard deviation were calculated. $P$ value less than 0.05 was considered significant.

\section{Results}

In this study, we recorded the demographic characteristics, clinical symptoms and the measurement of lesions of participating patients in the following Tables 1-4. 
Table 1. Demographics and clinical characteristics of studied samples.

\begin{tabular}{|c|c|c|c|c|}
\hline \multicolumn{2}{|c|}{ Variable } & HENE + Clindamycin & Clindamycin & $\mathrm{p}$ \\
\hline \multicolumn{2}{|c|}{ Age (year) } & $22.6 \pm 4.8$ & $21.7 \pm 5.1$ & $>0.05$ \\
\hline \multirow{2}{*}{ Sex } & Male & 4 & 4 & \multirow{2}{*}{$>0.05$} \\
\hline & Female & 27 & 26 & \\
\hline \multirow{2}{*}{ Severity } & Moderate & 20 & 20 & \multirow{2}{*}{$>0.05$} \\
\hline & Mild & 11 & 10 & \\
\hline
\end{tabular}

Table 2. Lesion areas on patients' body.

\begin{tabular}{ccc}
\hline Locations & Number of patients & $\%$ \\
\hline Face & 213 & 99.1 \\
Chest & 44 & 20.5 \\
Back & 70 & 32.6 \\
Others & 5 & 2.3 \\
\hline
\end{tabular}

Table 3. Acnes types and rate $(\mathrm{n}=215)$.

\begin{tabular}{ccc}
\hline Lesions & Number of patients & $\%$ \\
\hline Papule & 213 & 99.1 \\
Whitehead & 189 & 87.9 \\
Blackhead & 180 & 83.7 \\
Erythema & 172 & 80.0 \\
Pustule & 163 & 75.8 \\
Nodule & 17 & 7.9 \\
Cyst & 17 & 7.9 \\
Telangiectasia & 38 & 17.7 \\
Atrophic scar & 23 & 10.7 \\
Keloid & 3 & 1.4 \\
\hline
\end{tabular}

Table 4. Clinical symptoms $(\mathrm{n}=215)$.

\begin{tabular}{ccc}
\hline Symptoms & Number of patients & $\%$ \\
\hline Pruritus & 64 & 29.8 \\
Painful & 91 & 42.3 \\
Burning & 29 & 13.5 \\
No symptoms & 79 & 36.7
\end{tabular}

\subsection{Demographics and Clinical Symptoms before Treatments}

The age of HENE + Clindamycin and Clindamycin group had the mean \pm SD value of $22.6 \pm 4.8$ and $21.7 \pm 5.1$, respectively. Two groups are similar in sex and disease severity. 
More than $99 \%$ of our patients had acne on the face. $20.5 \%, 32.6 \%$ and $2.3 \%$ of patients had acne in the chest, back and other positions.

Papule was the lesion that more than $99 \%$ of our patients suffered from. Keloid was the least in our study with $1.4 \%$.

$29.8 \%, 42.3 \%$ and $13.5 \%$ of patients had symptoms like pruritus, pain and burning, consecutively.

\subsection{Compared Results from Weekly Treatments}

The following Tables 5-8 indicated the recovery progress from our weekly treatments.

After 1 -month treatment, $12.9 \%$ and $87.1 \%$ of patients in the HENE + Clindamycin group achieved good and moderate results, respectively. Meanwhile, in the Clindamycin group, only $6.7 \%$ reached good result. There was no clinical significant difference in efficacy between 2 groups $(\mathrm{p}>0.05)$.

Table 5. Comparison of results after 1-month treatment.

\begin{tabular}{cccccc}
\hline \multirow{2}{*}{ Levels } & \multicolumn{2}{c}{ HENE + Clindamycin } & \multicolumn{2}{c}{ Clindamycin } & $\mathrm{p}$ \\
\cline { 2 - 5 } Very good & $\mathrm{N}$ & $\%$ & $\mathrm{n}$ & $\%$ & \\
Good & 0 & 0 & 0 & 0 & $>0.05$ \\
Moderate & 4 & 12.9 & 2 & 6.7 & $\left(\chi^{2}=0.669\right)$ \\
Poor & 27 & 87.1 & 28 & 93.3 & 0 \\
Total & 0 & 0 & 0 & 100 & \\
\hline
\end{tabular}

Table 6. Comparison of results after 2-month treatment.

\begin{tabular}{cccccc}
\hline \multirow{2}{*}{ Levels } & \multicolumn{2}{c}{ HENE + Clindamycin } & \multicolumn{2}{c}{ Clindamycin } & $\mathrm{p}$ \\
\cline { 2 - 5 } & $\mathrm{N}$ & $\%$ & $\mathrm{n}$ & $\%$ & \\
Very good & 9 & 29.0 & 0 & 0 & \\
Good & 20 & 64.5 & 18 & 60.0 & $<0.05$ \\
Moderate & 2 & 6.5 & 12 & 40.0 & $\left(\chi^{2}=16.236\right)$ \\
Poor & 0 & 0 & 0 & 0 & 100.0
\end{tabular}

Table 7. Comparison of results after 3-month treatment.

\begin{tabular}{cccccc}
\hline \multirow{2}{*}{ Levels } & \multicolumn{2}{c}{ HENE + Clindamycin } & \multicolumn{2}{c}{ Clindamycin } & $\mathrm{p}$ \\
\cline { 2 - 5 } & $\mathrm{N}$ & $\%$ & $\mathrm{n}$ & $\%$ & \\
Very good & 16 & 51.6 & 9 & 30.0 & \\
Good & 15 & 48.4 & 15 & 50.0 & $<0.05$ \\
Moderate & 0 & 0 & 6 & 20.0 & $\left(\chi^{2}=7.946\right)$ \\
Poor & 0 & 0 & 0 & 0 & 100.0
\end{tabular}


Table 8. Comparison of adverse events after 3-month treatment.

\begin{tabular}{cccccc}
\hline \multirow{2}{*}{ Variable } & \multicolumn{2}{c}{ HENE + Clindamycin } & \multicolumn{2}{c}{ Clindamycin } & $\mathrm{p}$ \\
\cline { 2 - 4 } & $\mathrm{N}$ & $\%$ & $\mathrm{n}$ & $\%$ & \\
\hline Pruritus & 4 & 12.9 & 5 & 16.7 & \\
Erythema & 2 & 6.5 & 3 & 10.0 & $>0.05$ \\
Dryness & 1 & 3.2 & 1 & 3.3 & \\
Melasma & 0 & 0 & 0 & 0 & \\
\hline
\end{tabular}

After 2-month treatment, the percentage of patients in HENE + Clindamycin group acquiring very good result was $29.9 \%$. In contrast, no patients in another group earned the same outcome. The treatment efficacy is better with HENE + Clindamycin than Clindamycin alone $(\mathrm{p}<0.05)$.

After 3-month treatment, more than half of patients attained very good result. In the Clindamycin group, there were only $30 \%$ of patients gaining the same output. Treatment efficacy is better with HENE + Clindamycin than Clindamycin alone $(\mathrm{p}<0.05)$.

After 3 months of treatment, $12.9 \%, 6.5 \%$ and $3.2 \%$ of patients in HENE + Clindamycin group suffered from pruritus, erythema and dryness, respectively. In the meantime, $16.7 \%, 10 \%$ and $3.3 \%$ of patients in Clindamycin group had the same side effects. AEs during 3 months of treatment in two groups were similar, not significant, which allowed continuing treatments.

\section{Discussion}

Patients were randomized in 2 groups, with no difference in age, gender, or severity of disease with $p>0.05$. Therefore, the comparison of treatment results of the two groups is accurate and reliable. The results in Table 1 shows that after one month of treatment, the patients in the HENE + Clindamycin group and the Clindamycin group meets the moderate and good response rates of $87.1 \%$; $12.9 \%$, respectively; and 93.3\%; $6.7 \%$ respectively, none of whom achieved good response, the difference was not statistically significant with $\mathrm{p}>0.05$.

Results after 2 months of treatment in Table 2 show that the proportion of patients in the HENE + Clindamycin group was very good at $29.0 \%$, good $64.5 \%$, moderate $6.5 \%$, while patients in the Clindamycin group only achieved good or moderate level $\mathrm{p}<0.05$.

After 3 months of treatment, as shown in Table 3, 100\% of the patients in the $H E N E$ + Clindamycin group shows positive results, of which $51.6 \%$ are very good, $48.4 \%$ are good, and the Clindamycin group are very good-30.0\%, good- $50 \%$ and moderate $20 \%$; $p<0.05$.

Therefore, after 3 months of treatment, the efficacy of He-Ne laser treatment in combination with Clindamycin $1 \%$ is better than treatment with Clindamycin $1 \%$ alone $(\mathrm{p}<0.05)$.

According to Avci et al. (2013) [1], low-energy laser light therapy had been 
shown the efficacy in treating acne up to $70 \%$.

The explanation for the He-Ne laser treatment that effectively treats acne is the effect of the beam on the P. acne bacteria. The pacacillus metabolite produced porphyrin, including uroporphyrin, coproporphyrin III, and protoporphyrin IX, which has a strong absorbing potential with visible light at wavelengths of $400-700 \mathrm{~nm}$, in which the blue light is $415 \mathrm{~nm}$, red light $632.8 \mathrm{~nm}$ as an endogenous light sensitive substance [2] [5]. The light-absorbing process causes a photochemical reaction that activates free radicals to react and releases millions of oxygen atoms in the hair follicle to kill the P. acnes bacteria without damaging the normal tissues [2]. In addition, the $632.8 \mathrm{~nm}$ wavelength also has the ability to penetrate deep into the tissues, directly affecting sebaceous secretion of the sebaceous gland, affecting keratinocyte cells, macrophages and some other cells producing the cytokine, stimulating the growth of fibroblasts and the product of the growth factors; thereby affecting the inflammatory process, healing the wound [5].

The results in Table 5, AEs in 2 groups are similar and include mainly pruritus, erythema, dryness. All of AEs are mild or moderate. The limitation of this study is that the severity of our patients is only mild and moderate, our sample size is not large and relapse is not evaluated. There is a need for further randomized control trials accessing the efficacy and safety of He-Ne laser in the treatment of acne vulgaris.

\section{Conclusions}

- Combination of He-Ne laser and clindamycin is effective in the treatment of mild to moderate acne: very good $51.6 \%$, good $48.4 \%$.

- Combination of He-Ne laser and clindamycin is better than clindamycin alone in treating normal and mild acne.

\section{Conflicts of Interest}

The authors declare no conflicts of interest regarding the publication of this paper.

\section{References}

[1] Avci, P., Gupta, A., Sadasivan, M., et al. (2013) Low-Level Laser (Light) Therapy (LLLT) in Skin: Stimulating, Healinh, Restoring. Seminars in Cutaneous Medicine and Surgery, 32, 41-52.

[2] Arsiwala, S.Z. (2016) Laser and Light Treatment of Acne. Textbook of Laser in Dermatology, The Health Sciences Publisher, New Delhi, 134-142.

https://doi.org/10.5005/jp/books/12874_19

[3] Minh, N.T. (2002) Cause of Acne. Dermatological Updates, 1, 43-45.

[4] Wolff, K. and Johnson, R.A. (2013) Acne vulgaris (Common Acne) and Cystic Acne. Fitzpatrick's Color Atlas and Synopsis of Clinical Dermatology. 7th Edition, McGraw-Hill, New York, 2-7.

[5] Sardari, F. and Ahrari, F. (2016) The Effect of Low-Level Helium-Neon Laser on Wound Healing. Dental Research Journal, 13, 24-29. https://doi.org/10.4103/1735-3327.174693 